STRUCTURE
EEPORTS

OPEN $\curvearrowright$ ACCESS

ISSN 1600-5368

\section{Crystal structure of cis-1-(2-methyl-} 1,2,3,4-tetrahydroquinolin-4-yl)azepan2-one

\author{
P. S. Pradeep, ${ }^{\text {a }}$ S. Naveen, ${ }^{\text {b }}$ M. N. Kumara, ${ }^{c}$ \\ K. M. Mahadevan ${ }^{\mathrm{a}}$ and N. K. Lokanath ${ }^{\mathrm{d} *}$
}

a Department of Chemistry, Kuvempu University, Jnanasahyadri, Shankaraghatta 577 451, India, 'bnstitution of Excellence, University of Mysore, Manasagangotri, Mysore 570 006, India, 'Department of Chemistry, Yuvaraja's College, University of Mysore, Mysore 570 005, India, and d Department of Studies in Physics, University of Mysore, Manasagangotri, Mysore 570 006, India. *Correspondence e-mail:

lokanath@physics.uni-mysore.ac.in

Received 24 July 2014; accepted 2 August 2014

Edited by D.-J. Xu, Zhejiang University (Yuquan Campus), China

In the title compound, $\mathrm{C}_{16} \mathrm{H}_{22} \mathrm{~N}_{2} \mathrm{O}$, the azepan-2-one ring adopts a chair conformation, while the 1,2,3,4-tetrahydropyridine ring adopts a half-chair conformation. In the crystal, molecules are linked by $\mathrm{N}-\mathrm{H} \cdots \mathrm{O}$ hydrogen bonds, forming

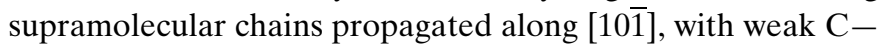
$\mathrm{H}$... O interactions occurring between the chains.

Keywords: crystal structure; tetrahydroquinolines; azepan-2-one; hydrogen bonding.

CCDC reference: 1017682

\section{Related literature}

For applications of tetrahydroquinolines, see: Konishi et al. (1990).

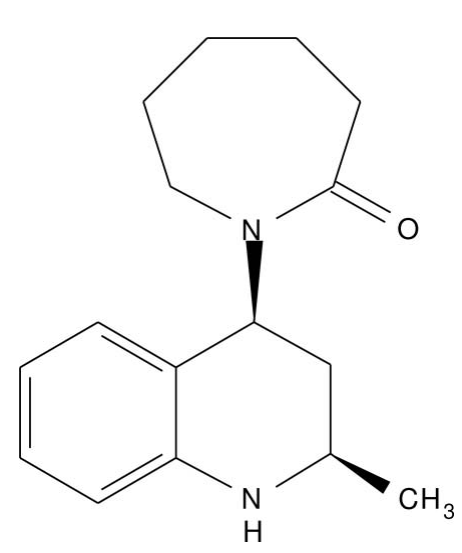

\section{Experimental}

2.1. Crystal data
$\mathrm{C}_{16} \mathrm{H}_{22} \mathrm{~N}_{2} \mathrm{O}$

$M_{r}=258.36$

Monoclinic, $P 2_{1} / n$

$a=9.1640(17) \AA$

$b=13.1687(18) \AA$

$c=11.988(2) \AA$

$\beta=96.825(11)^{\circ}$

\subsection{Data collection}

Bruker X8 Proteum diffractometer Absorption correction: multi-scan (SADABS; Bruker, 2013)

$T_{\min }=0.874, T_{\max }=0.884$

\subsection{Refinement}

$R\left[F^{2}>2 \sigma\left(F^{2}\right)\right]=0.049$

$w R\left(F^{2}\right)=0.139$

$S=1.06$

2343 reflections

$$
\begin{aligned}
& V=1436.4(4) \AA^{3} \\
& Z=4 \\
& C u K \alpha \text { radiation } \\
& \mu=0.59 \mathrm{~mm}^{-1} \\
& T=296 \mathrm{~K} \\
& 0.23 \times 0.22 \times 0.21 \mathrm{~mm}
\end{aligned}
$$

Table 1

Hydrogen-bond geometry $\left(\AA{ }^{\circ}\right)$.

\begin{tabular}{lllll}
\hline$D-\mathrm{H} \cdots A$ & $D-\mathrm{H}$ & $\mathrm{H} \cdots A$ & $D \cdots A$ & $D-\mathrm{H} \cdots A$ \\
\hline $\mathrm{N} 1-\mathrm{H} 1 \cdots \mathrm{O} 19^{\mathrm{i}}$ & 0.86 & 2.40 & $2.988(2)$ & 126 \\
$\mathrm{C} 14-\mathrm{H} 14 A \cdots \mathrm{O} 19^{\mathrm{ii}}$ & 0.97 & 2.57 & $3.320(2)$ & 134 \\
\hline
\end{tabular}

Symmetry codes: (i) $x+\frac{1}{2},-y+\frac{3}{2}, z-\frac{1}{2}$; (ii) $-x+\frac{1}{2}, y+\frac{1}{2},-z+\frac{1}{2}$.

Data collection: APEX2 (Bruker, 2013); cell refinement: SAINT (Bruker, 2013); data reduction: $S A I N T$; $\operatorname{program}(\mathrm{s})$ used to solve structure: SHELXS97 (Sheldrick, 2008); program(s) used to refine structure: SHELXL97 (Sheldrick, 2008); molecular graphics: Mercury (Macrae et al., 2008); software used to prepare material for publication: Mercury.

\section{Acknowledgements}

The authors are thankful to IOE, Vijnana Bhavana, University of Mysore, Mysore, for providing the single-crystal X-ray diffractometer facility. 


\section{data reports}

Supporting information for this paper is available from the $\mathrm{IUCr}$ electronic archives (Reference: XU5808).

\section{References}

Bruker (2013). APEX2, SAINT and SADABS. Bruker AXS Inc., Madison, Wisconsin, USA.
Konishi, M., Ohkuma, H., Tsuno, T. \& Oki, T. (1990). J. Am. Chem. Soc. 112, $3715-3716$.

Macrae, C. F., Bruno, I. J., Chisholm, J. A., Edgington, P. R., McCabe, P., Pidcock, E., Rodriguez-Monge, L., Taylor, R., van de Streek, J. \& Wood, P. A. (2008). J. Appl. Cryst. 41, 466-470.

Sheldrick, G. M. (2008). Acta Cryst. A64, 112-122. 


\section{supporting information}

Acta Cryst. (2014). E70, o981-o982［doi:10.1107/S1600536814017826]

\section{Crystal structure of cis-1-(2-methyl-1,2,3,4-tetrahydroquinolin-4-yl)azepan-2- one}

\section{P. S. Pradeep, S. Naveen, M. N. Kumara, K. M. Mahadevan and N. K. Lokanath}

\section{S1. Comment}

Tetrahydroquinolines are an important family of heterocyclic compounds having wide range of biological activities which includes antimalarial, antitumoral, antioxidant, etc. In particular 2-Methyl-1,2,3,4-tetrahydroquinoline is present in human brain and a natural antitumor antibiotic, has a complex structure built on the tetrahydroquinoline system (Konishi et al., 1990). Hence, in continuation of our effort to identify new quinoline based therapeutic agents, the title compound has been synthesized and herein we report its crystal structure.

The ORTEP of the molecule is shown in figure 1. The title compound is chiral. In the arbitrarily chosen asymmetric molecule, $\mathrm{C} 2$ has $S$ configuration whereas $\mathrm{C} 4$ has $R$ configuration. The azepan ring lies in the equatorial plane of the fused rings as indicated by the dihedral angle value of $66.28(8)^{\circ}$. A study of torsion angles, asymmetric parameters and least squares plane calculations reveals that the quinoline ring in the structure adopts a half chair conformation with the atom C3 deviating by 0.3278 (18) $\AA$ from the least-squares plane defined by the atoms $\mathrm{N} 1 / \mathrm{C} 2 / \mathrm{C} 3 / \mathrm{C} 4 / \mathrm{C} 5 / \mathrm{C} 10$. This is confirmed by the puckering amplitude $Q=0.4857$ (18) $\AA$. The structure exhibits weak intermolecular hydrogen bonds of the type $\mathrm{C}-\mathrm{H} \cdots \mathrm{O}$ and $\mathrm{N}-\mathrm{H} \cdots \mathrm{O}$. The packing of the molecules when viewed along the $a$ axis indicate that they are stacked in pairs.

\section{S2. Experimental}

A catalytic amount of $\mathrm{SbF}_{3}(10 \mathrm{~mol} \%)$ was added to the mixture of aniline(1 equivalent) and $N$-vinyl caprolactam(2-3 equivalent) in acetonitrile $(5-10 \mathrm{ml})$. The reaction mixture was stirred at ambient temperature $\left(\sim 25^{\circ} \mathrm{C}\right)$ for $20-70 \mathrm{~min}$. The reaction was monitored by TLC by using ethyl acetate/hexane as eluent. After the completion of the reaction, the solvent was removed under vacuo. The crude product was then quenched with water and the catalyst was decomposed by addition of appropriate amount of sodium bicarbonate solution, extracted with ethyl acetate $(10 \mathrm{ml} \times 5$ times $)$, dried and was purified by column chromatography using ethyl acetate/hexane as eluent (pet ether/ethyl acetate 80:20 v/v). The white solid crystals were obtained by slow evaporation method by using petroleum ether: ethyl acetate, $8: 2 v / v$ as solvents. M.P. $=155-160^{\circ} \mathrm{C}$. Yield: $93 \%$.

\section{S3. Refinement}

The hydrogen atoms were placed geometrically with $\mathrm{N}-\mathrm{H}=0.86, \mathrm{C}-\mathrm{H}=0.93-0.98 \AA$, and allowed to ride on their parent atoms with $U_{\text {iso }}(\mathrm{H})=1.5 U_{\text {eq }}(\mathrm{C})$ for the methyl $\mathrm{H}$ atoms and $1.2 U_{\mathrm{eq}}(\mathrm{N}, \mathrm{C})$ for the others. 


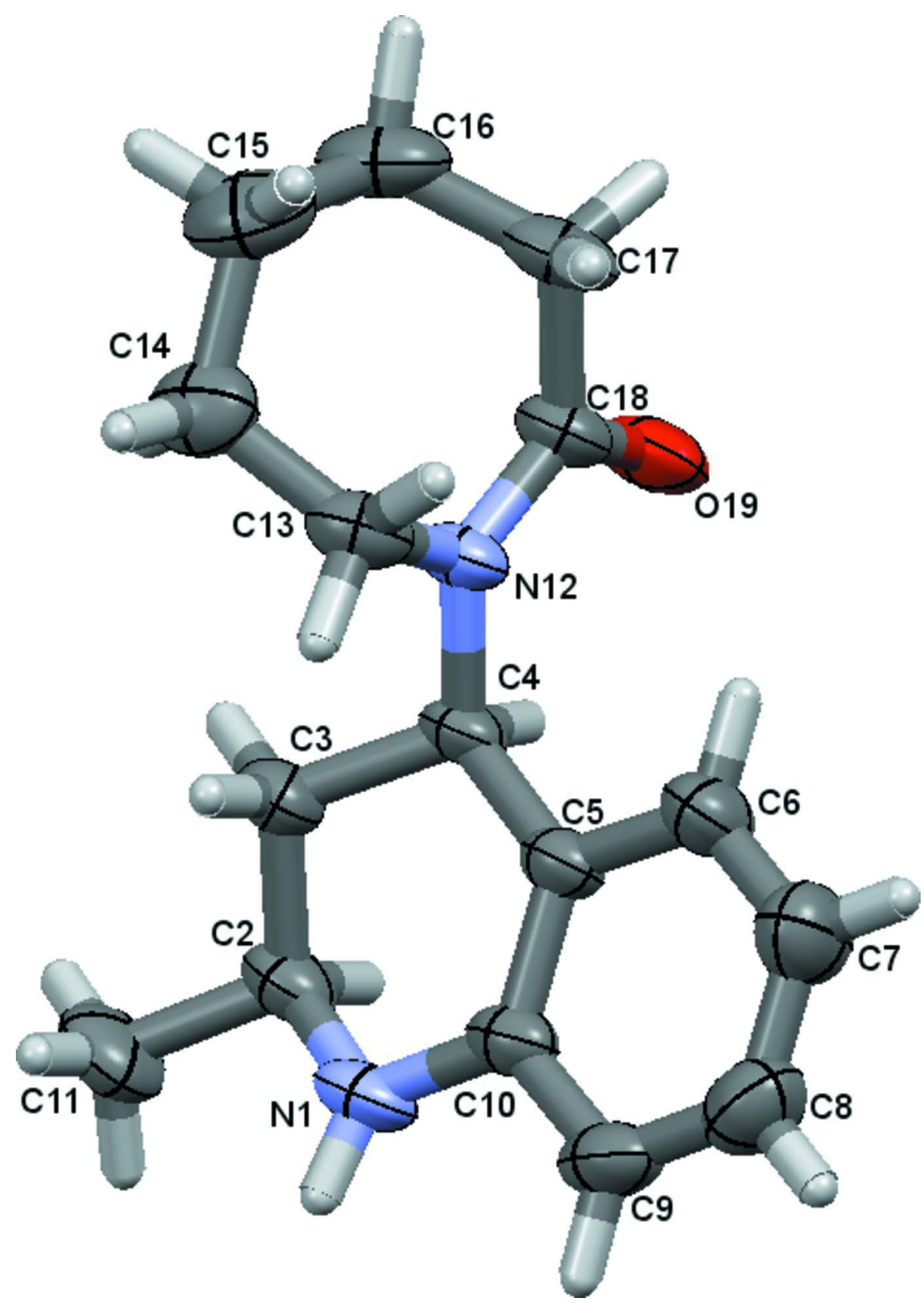

\section{Figure 1}

A view of the title molecule, with atom labelling. Displacement ellipsoids are drawn at the $50 \%$ probability level. 


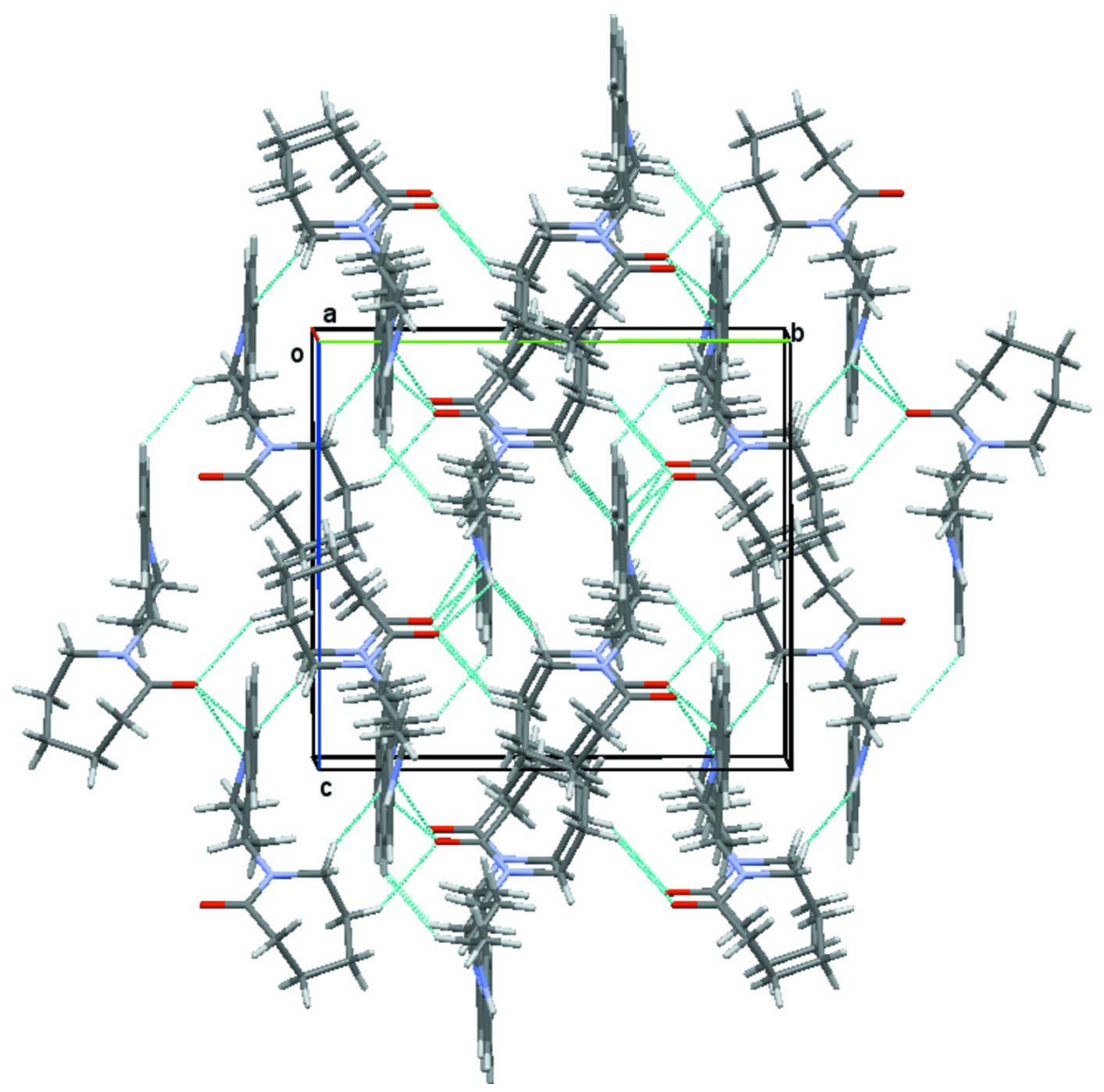

Figure 2

A viewed along the $a$ axis of the crystal packing of the title compound.

\section{1-(2-Methyl-1,2,3,4-tetrahydroquinolin-4-yl)azepan-2-one}

\section{Crystal data}

$\mathrm{C}_{16} \mathrm{H}_{22} \mathrm{~N}_{2} \mathrm{O}$

$M_{r}=258.36$

Monoclinic, $P 2_{1} / n$

Hall symbol: -P 2yn

$a=9.1640(17) \AA$

$b=13.1687(18) \AA$

$c=11.988(2) \AA$

$\beta=96.825(11)^{\circ}$

$V=1436.4(4) \AA^{3}$

$Z=4$

\section{Data collection}

Bruker X8 Proteum diffractometer

Radiation source: Bruker MicroStar microfocus rotating anode

Helios multilayer optics monochromator

Detector resolution: 18.4 pixels $\mathrm{mm}^{-1}$
$F(000)=560$

$D_{\mathrm{x}}=1.195 \mathrm{Mg} \mathrm{m}^{-3}$

$\mathrm{Cu} K \alpha$ radiation, $\lambda=1.54178 \AA$

Cell parameters from 2343 reflections

$\theta=6.7-64.8^{\circ}$

$\mu=0.59 \mathrm{~mm}^{-1}$

$T=296 \mathrm{~K}$

Block, colorless

$0.23 \times 0.22 \times 0.21 \mathrm{~mm}$

$\varphi$ and $\omega$ scans

Absorption correction: multi-scan

(SADABS; Bruker, 2013)

$T_{\min }=0.874, T_{\max }=0.884$

7903 measured reflections

2343 independent reflections 
2106 reflections with $I>2 \sigma(I)$

$R_{\text {int }}=0.028$

$\theta_{\max }=64.8^{\circ}, \theta_{\min }=6.7^{\circ}$

\section{Refinement}

Refinement on $F^{2}$

Least-squares matrix: full

$R\left[F^{2}>2 \sigma\left(F^{2}\right)\right]=0.049$

$w R\left(F^{2}\right)=0.139$

$S=1.06$

2343 reflections

173 parameters

0 restraints

Primary atom site location: structure-invariant direct methods $h=-10 \rightarrow 10$
$k=-15 \rightarrow 4$
$l=-14 \rightarrow 13$

Secondary atom site location: difference Fourier map

Hydrogen site location: inferred from neighbouring sites

$\mathrm{H}$-atom parameters constrained

$w=1 /\left[\sigma^{2}\left(F_{\mathrm{o}}^{2}\right)+(0.0671 P)^{2}+0.4283 P\right]$

where $P=\left(F_{\mathrm{o}}^{2}+2 F_{\mathrm{c}}^{2}\right) / 3$

$(\Delta / \sigma)_{\max }<0.001$

$\Delta \rho_{\max }=0.34 \mathrm{e} \AA^{-3}$

$\Delta \rho_{\min }=-0.36$ e $\AA^{-3}$

\section{Special details}

Geometry. Bond distances, angles etc. have been calculated using the rounded fractional coordinates. All su's are estimated from the variances of the (full) variance-covariance matrix. The cell e.s.d.'s are taken into account in the estimation of distances, angles and torsion angles

Refinement. Refinement on $F^{2}$ for ALL reflections except those flagged by the user for potential systematic errors. Weighted $R$-factors $w R$ and all goodnesses of fit $S$ are based on $F^{2}$, conventional $R$-factors $R$ are based on $F$, with $F$ set to zero for negative $F^{2}$. The observed criterion of $F^{2}>\sigma\left(F^{2}\right)$ is used only for calculating - $R$-factor-obs etc. and is not relevant to the choice of reflections for refinement. $R$-factors based on $F^{2}$ are statistically about twice as large as those based on $F$, and $R$-factors based on ALL data will be even larger.

Fractional atomic coordinates and isotropic or equivalent isotropic displacement parameters $\left(\AA^{2}\right)$

\begin{tabular}{|c|c|c|c|c|}
\hline & $x$ & $y$ & $z$ & $U_{\text {iso }} * / U_{\text {eq }}$ \\
\hline O19 & $0.20029(15)$ & $0.75502(10)$ & $0.32245(11)$ & $0.0661(5)$ \\
\hline N1 & $0.53573(16)$ & $0.85016(11)$ & $-0.01036(12)$ & $0.0538(5)$ \\
\hline N12 & $0.28345(14)$ & $0.90157(10)$ & $0.25474(10)$ & $0.0438(4)$ \\
\hline $\mathrm{C} 2$ & $0.60480(18)$ & $0.82217(14)$ & $0.10008(15)$ & $0.0511(6)$ \\
\hline $\mathrm{C} 3$ & $0.52791(17)$ & $0.87701(14)$ & $0.18769(14)$ & $0.0497(5)$ \\
\hline $\mathrm{C} 4$ & $0.36664(17)$ & $0.84597(12)$ & $0.17675(13)$ & $0.0421(5)$ \\
\hline $\mathrm{C} 5$ & $0.29631(17)$ & $0.85491(12)$ & $0.05626(13)$ & $0.0439(5)$ \\
\hline C6 & $0.1450(2)$ & $0.86076(16)$ & $0.03030(16)$ & $0.0593(6)$ \\
\hline $\mathrm{C} 7$ & $0.0790(2)$ & 0.86425 (19) & $-0.07861(17)$ & $0.0718(8)$ \\
\hline $\mathrm{C} 8$ & 0.1649 & $0.86264(17)$ & $-0.16454(16)$ & $0.0700(7)$ \\
\hline C9 & $0.3153(2)$ & $0.85678(14)$ & $-0.14226(15)$ & $0.0605(7)$ \\
\hline $\mathrm{C} 10$ & 0.38380 (19) & $0.85289(12)$ & $-0.03180(14)$ & $0.0459(5)$ \\
\hline $\mathrm{C} 11$ & $0.7676(2)$ & 0.84612 (19) & $0.1097(2)$ & $0.0743(8)$ \\
\hline $\mathrm{C} 13$ & 0.28372 (19) & 1.01269 (13) & $0.25122(14)$ & $0.0514(6)$ \\
\hline $\mathrm{C} 14$ & $0.3621(2)$ & $1.06237(15)$ & $0.35552(17)$ & $0.0640(7)$ \\
\hline C15 & $0.2718(3)$ & $1.06735(17)$ & $0.45383(18)$ & $0.0770(8)$ \\
\hline C16 & $0.2169(3)$ & $0.96542(17)$ & $0.49058(17)$ & $0.0720(8)$ \\
\hline $\mathrm{C} 17$ & $0.1190(2)$ & $0.90829(16)$ & $0.40028(17)$ & $0.0627(7)$ \\
\hline $\mathrm{C} 18$ & $0.20364(18)$ & $0.84820(14)$ & $0.32180(13)$ & $0.0486(6)$ \\
\hline H1 & 0.58880 & 0.86510 & -0.06260 & $0.0640^{*}$ \\
\hline $\mathrm{H} 2$ & 0.59290 & 0.74890 & 0.10990 & $0.0610^{*}$ \\
\hline $\mathrm{H} 3 \mathrm{~A}$ & 0.53550 & 0.94980 & 0.17750 & $0.0600^{*}$ \\
\hline
\end{tabular}




$\begin{array}{lllll}\text { H3B } & 0.57490 & 0.86010 & 0.26220 & 0.0600^{*} \\ \text { H4 } & 0.36380 & 0.77400 & 0.19710 & 0.0510^{*} \\ \text { H6 } & 0.08640 & 0.86240 & 0.08850 & 0.0710^{*} \\ \text { H7 } & -0.02280 & 0.86770 & -0.09380 & 0.0860^{*} \\ \text { H8 } & 0.12120 & 0.86550 & -0.23860 & 0.0840^{*} \\ \text { H9 } & 0.37220 & 0.85540 & -0.20150 & 0.0730^{*} \\ \text { H11A } & 0.81160 & 0.80920 & 0.05330 & 0.1120^{*} \\ \text { H11B } & 0.81330 & 0.82670 & 0.18280 & 0.1120^{*} \\ \text { H11C } & 0.78080 & 0.91760 & 0.09920 & 0.1120^{*} \\ \text { H13A } & 0.18280 & 1.03640 & 0.24060 & 0.0620^{*} \\ \text { H13B } & 0.33020 & 1.03440 & 0.18670 & 0.0620^{*} \\ \text { H14A } & 0.38960 & 1.13080 & 0.33670 & 0.0770^{*} \\ \text { H14B } & 0.45190 & 1.02500 & 0.37870 & 0.0770^{*} \\ \text { H15A } & 0.33110 & 1.09830 & 0.51720 & 0.0920^{*} \\ \text { H15B } & 0.18770 & 1.11120 & 0.43340 & 0.0920^{*} \\ \text { H16A } & 0.16270 & 0.97610 & 0.55430 & 0.0860^{*} \\ \text { H16B } & 0.30120 & 0.92320 & 0.51580 & 0.0860^{*} \\ \text { H17A } & 0.05640 & 0.86230 & 0.43620 & 0.0750^{*} \\ \text { H17B } & 0.05600 & 0.95660 & 0.35650 & 0.0750^{*}\end{array}$

Atomic displacement parameters $\left(\AA^{2}\right)$

\begin{tabular}{lllllll}
\hline & $U^{11}$ & $U^{22}$ & $U^{33}$ & $U^{12}$ & $U^{13}$ & $U^{23}$ \\
\hline O19 & $0.0806(9)$ & $0.0577(9)$ & $0.0692(9)$ & $-0.0120(7)$ & $0.0477(7)$ & $-0.0006(6)$ \\
N1 & $0.0525(8)$ & $0.0624(9)$ & $0.0526(8)$ & $0.0030(7)$ & $0.0321(7)$ & $0.0032(7)$ \\
N12 & $0.0447(7)$ & $0.0501(8)$ & $0.0406(7)$ & $0.0023(6)$ & $0.0221(6)$ & $0.0023(6)$ \\
C2 & $0.0446(9)$ & $0.0534(10)$ & $0.0599(10)$ & $0.0014(7)$ & $0.0254(8)$ & $0.0009(8)$ \\
C3 & $0.0411(8)$ & $0.0607(10)$ & $0.0501(9)$ & $-0.0022(7)$ & $0.0173(7)$ & $-0.0045(8)$ \\
C4 & $0.0419(8)$ & $0.0472(9)$ & $0.0412(8)$ & $-0.0003(7)$ & $0.0212(6)$ & $0.0003(7)$ \\
C5 & $0.0441(9)$ & $0.0469(9)$ & $0.0436(9)$ & $-0.0039(7)$ & $0.0179(7)$ & $-0.0008(7)$ \\
C6 & $0.0468(10)$ & $0.0805(13)$ & $0.0529(10)$ & $-0.0060(9)$ & $0.0161(8)$ & $0.0002(9)$ \\
C7 & $0.0567(11)$ & $0.0964(16)$ & $0.0609(12)$ & $-0.0073(11)$ & $0.0017(9)$ & $0.0001(11)$ \\
C8 & $0.0819(14)$ & $0.0791(14)$ & $0.0470(10)$ & $-0.0082(11)$ & $0.0000(10)$ & $-0.0047(9)$ \\
C9 & $0.0812(14)$ & $0.0618(11)$ & $0.0424(9)$ & $-0.0068(9)$ & $0.0238(9)$ & $-0.0057(8)$ \\
C10 & $0.0555(9)$ & $0.0415(9)$ & $0.0448(9)$ & $-0.0023(7)$ & $0.0227(7)$ & $-0.0012(7)$ \\
C11 & $0.0451(10)$ & $0.0932(16)$ & $0.0900(15)$ & $0.0016(10)$ & $0.0301(10)$ & $-0.0044(12)$ \\
C13 & $0.0576(10)$ & $0.0523(10)$ & $0.0480(9)$ & $0.0097(8)$ & $0.0219(8)$ & $0.0091(7)$ \\
C14 & $0.0803(13)$ & $0.0496(11)$ & $0.0641(12)$ & $0.0029(9)$ & $0.0170(10)$ & $-0.0019(9)$ \\
C15 & $0.1127(18)$ & $0.0636(13)$ & $0.0579(12)$ & $0.0138(12)$ & $0.0233(12)$ & $-0.0091(10)$ \\
C16 & $0.0985(16)$ & $0.0738(14)$ & $0.0498(11)$ & $0.0185(12)$ & $0.0348(10)$ & $-0.0001(9)$ \\
C17 & $0.0607(11)$ & $0.0735(13)$ & $0.0616(11)$ & $0.0111(9)$ & $0.0388(9)$ & $0.0080(10)$ \\
C18 & $0.0454(9)$ & $0.0591(11)$ & $0.0454(9)$ & $0.0002(7)$ & $0.0225(7)$ & $0.0031(7)$ \\
& & & & & & \\
\hline
\end{tabular}

Geometric parameters $\left(\AA,{ }^{\circ}\right)$

\begin{tabular}{llll}
\hline $\mathrm{O} 19-\mathrm{C} 18$ & $1.228(2)$ & $\mathrm{C} 2-\mathrm{H} 2$ & 0.9800 \\
$\mathrm{~N} 1-\mathrm{C} 2$ & $1.446(2)$ & $\mathrm{C} 3-\mathrm{H} 3 \mathrm{~A}$ & 0.9700 \\
$\mathrm{~N} 1-\mathrm{C} 10$ & $1.386(2)$ & $\mathrm{C} 3-\mathrm{H} 3 \mathrm{~B}$ & 0.9700
\end{tabular}




\begin{tabular}{|c|c|c|c|}
\hline $\mathrm{N} 12-\mathrm{C} 4$ & $1.470(2)$ & $\mathrm{C} 4-\mathrm{H} 4$ & 0.9800 \\
\hline $\mathrm{N} 12-\mathrm{C} 13$ & $1.464(2)$ & $\mathrm{C} 6-\mathrm{H} 6$ & 0.9300 \\
\hline $\mathrm{N} 12-\mathrm{C} 18$ & $1.347(2)$ & $\mathrm{C} 7-\mathrm{H} 7$ & 0.9300 \\
\hline $\mathrm{N} 1-\mathrm{H} 1$ & 0.8600 & $\mathrm{C} 8-\mathrm{H} 8$ & 0.9300 \\
\hline $\mathrm{C} 2-\mathrm{C} 11$ & $1.516(3)$ & $\mathrm{C} 9-\mathrm{H} 9$ & 0.9300 \\
\hline $\mathrm{C} 2-\mathrm{C} 3$ & $1.516(2)$ & $\mathrm{C} 11-\mathrm{H} 11 \mathrm{~A}$ & 0.9600 \\
\hline $\mathrm{C} 3-\mathrm{C} 4$ & $1.524(2)$ & $\mathrm{C} 11-\mathrm{H} 11 \mathrm{~B}$ & 0.9600 \\
\hline $\mathrm{C} 4-\mathrm{C} 5$ & $1.515(2)$ & $\mathrm{C} 11-\mathrm{H} 11 \mathrm{C}$ & 0.9600 \\
\hline $\mathrm{C} 5-\mathrm{C} 6$ & $1.387(2)$ & $\mathrm{C} 13-\mathrm{H} 13 \mathrm{~A}$ & 0.9700 \\
\hline $\mathrm{C} 5-\mathrm{C} 10$ & $1.400(2)$ & C13-H13B & 0.9700 \\
\hline $\mathrm{C} 6-\mathrm{C} 7$ & $1.373(3)$ & $\mathrm{C} 14-\mathrm{H} 14 \mathrm{~A}$ & 0.9700 \\
\hline $\mathrm{C} 7-\mathrm{C} 8$ & $1.369(3)$ & $\mathrm{C} 14-\mathrm{H} 14 \mathrm{~B}$ & 0.9700 \\
\hline $\mathrm{C} 8-\mathrm{C} 9$ & $1.375(3)$ & $\mathrm{C} 15-\mathrm{H} 15 \mathrm{~A}$ & 0.9700 \\
\hline $\mathrm{C} 9-\mathrm{C} 10$ & $1.397(2)$ & C15-H15B & 0.9700 \\
\hline $\mathrm{C} 13-\mathrm{C} 14$ & $1.515(3)$ & $\mathrm{C} 16-\mathrm{H} 16 \mathrm{~A}$ & 0.9700 \\
\hline $\mathrm{C} 14-\mathrm{C} 15$ & $1.520(3)$ & $\mathrm{C} 16-\mathrm{H} 16 \mathrm{~B}$ & 0.9700 \\
\hline $\mathrm{C} 15-\mathrm{C} 16$ & $1.517(3)$ & C17-H17A & 0.9700 \\
\hline $\mathrm{C} 16-\mathrm{C} 17$ & $1.521(3)$ & C17-H17B & 0.9700 \\
\hline $\mathrm{C} 17-\mathrm{C} 18$ & $1.512(3)$ & & \\
\hline $\mathrm{C} 2-\mathrm{N} 1-\mathrm{C} 10$ & $119.82(14)$ & $\mathrm{C} 5-\mathrm{C} 4-\mathrm{H} 4$ & 107.00 \\
\hline $\mathrm{C} 4-\mathrm{N} 12-\mathrm{C} 13$ & $118.48(12)$ & $\mathrm{C} 5-\mathrm{C} 6-\mathrm{H} 6$ & 119.00 \\
\hline $\mathrm{C} 4-\mathrm{N} 12-\mathrm{C} 18$ & $118.62(13)$ & $\mathrm{C} 7-\mathrm{C} 6-\mathrm{H} 6$ & 119.00 \\
\hline $\mathrm{C} 13-\mathrm{N} 12-\mathrm{C} 18$ & $122.79(14)$ & $\mathrm{C} 6-\mathrm{C} 7-\mathrm{H} 7$ & 120.00 \\
\hline $\mathrm{C} 2-\mathrm{N} 1-\mathrm{H} 1$ & 120.00 & $\mathrm{C} 8-\mathrm{C} 7-\mathrm{H} 7$ & 120.00 \\
\hline $\mathrm{C} 10-\mathrm{N} 1-\mathrm{H} 1$ & 120.00 & $\mathrm{C} 7-\mathrm{C} 8-\mathrm{H} 8$ & 120.00 \\
\hline $\mathrm{C} 3-\mathrm{C} 2-\mathrm{C} 11$ & $112.49(16)$ & $\mathrm{C} 9-\mathrm{C} 8-\mathrm{H} 8$ & 120.00 \\
\hline $\mathrm{N} 1-\mathrm{C} 2-\mathrm{C} 3$ & $108.91(14)$ & $\mathrm{C} 8-\mathrm{C} 9-\mathrm{H} 9$ & 120.00 \\
\hline $\mathrm{N} 1-\mathrm{C} 2-\mathrm{C} 11$ & $109.66(16)$ & $\mathrm{C} 10-\mathrm{C} 9-\mathrm{H} 9$ & 120.00 \\
\hline $\mathrm{C} 2-\mathrm{C} 3-\mathrm{C} 4$ & $109.79(14)$ & $\mathrm{C} 2-\mathrm{C} 11-\mathrm{H} 11 \mathrm{~A}$ & 109.00 \\
\hline $\mathrm{N} 12-\mathrm{C} 4-\mathrm{C} 5$ & $111.95(13)$ & $\mathrm{C} 2-\mathrm{C} 11-\mathrm{H} 11 \mathrm{~B}$ & 109.00 \\
\hline $\mathrm{C} 3-\mathrm{C} 4-\mathrm{C} 5$ & $111.08(13)$ & $\mathrm{C} 2-\mathrm{C} 11-\mathrm{H} 11 \mathrm{C}$ & 109.00 \\
\hline $\mathrm{N} 12-\mathrm{C} 4-\mathrm{C} 3$ & $112.50(13)$ & $\mathrm{H} 11 \mathrm{~A}-\mathrm{C} 11-\mathrm{H} 11 \mathrm{~B}$ & 110.00 \\
\hline $\mathrm{C} 6-\mathrm{C} 5-\mathrm{C} 10$ & $118.62(15)$ & $\mathrm{H} 11 \mathrm{~A}-\mathrm{C} 11-\mathrm{H} 11 \mathrm{C}$ & 110.00 \\
\hline $\mathrm{C} 4-\mathrm{C} 5-\mathrm{C} 6$ & $121.27(15)$ & $\mathrm{H} 11 \mathrm{~B}-\mathrm{C} 11-\mathrm{H} 11 \mathrm{C}$ & 109.00 \\
\hline $\mathrm{C} 4-\mathrm{C} 5-\mathrm{C} 10$ & $120.05(14)$ & $\mathrm{N} 12-\mathrm{C} 13-\mathrm{H} 13 \mathrm{~A}$ & 109.00 \\
\hline $\mathrm{C} 5-\mathrm{C} 6-\mathrm{C} 7$ & $122.08(17)$ & $\mathrm{N} 12-\mathrm{C} 13-\mathrm{H} 13 \mathrm{~B}$ & 109.00 \\
\hline $\mathrm{C} 6-\mathrm{C} 7-\mathrm{C} 8$ & $119.17(19)$ & $\mathrm{C} 14-\mathrm{C} 13-\mathrm{H} 13 \mathrm{~A}$ & 109.00 \\
\hline $\mathrm{C} 7-\mathrm{C} 8-\mathrm{C} 9$ & $120.51(18)$ & $\mathrm{C} 14-\mathrm{C} 13-\mathrm{H} 13 \mathrm{~B}$ & 109.00 \\
\hline $\mathrm{C} 8-\mathrm{C} 9-\mathrm{C} 10$ & $120.89(17)$ & $\mathrm{H} 13 \mathrm{~A}-\mathrm{C} 13-\mathrm{H} 13 \mathrm{~B}$ & 108.00 \\
\hline $\mathrm{N} 1-\mathrm{C} 10-\mathrm{C} 5$ & $120.90(15)$ & $\mathrm{C} 13-\mathrm{C} 14-\mathrm{H} 14 \mathrm{~A}$ & 109.00 \\
\hline $\mathrm{N} 1-\mathrm{C} 10-\mathrm{C} 9$ & $120.33(16)$ & $\mathrm{C} 13-\mathrm{C} 14-\mathrm{H} 14 \mathrm{~B}$ & 109.00 \\
\hline $\mathrm{C} 5-\mathrm{C} 10-\mathrm{C} 9$ & $118.75(16)$ & $\mathrm{C} 15-\mathrm{C} 14-\mathrm{H} 14 \mathrm{~A}$ & 109.00 \\
\hline $\mathrm{N} 12-\mathrm{C} 13-\mathrm{C} 14$ & $114.22(14)$ & C15-C14-H14B & 109.00 \\
\hline $\mathrm{C} 13-\mathrm{C} 14-\mathrm{C} 15$ & $114.23(16)$ & $\mathrm{H} 14 \mathrm{~A}-\mathrm{C} 14-\mathrm{H} 14 \mathrm{~B}$ & 108.00 \\
\hline $\mathrm{C} 14-\mathrm{C} 15-\mathrm{C} 16$ & $114.64(18)$ & $\mathrm{C} 14-\mathrm{C} 15-\mathrm{H} 15 \mathrm{~A}$ & 109.00 \\
\hline $\mathrm{C} 15-\mathrm{C} 16-\mathrm{C} 17$ & $114.77(18)$ & $\mathrm{C} 14-\mathrm{C} 15-\mathrm{H} 15 \mathrm{~B}$ & 109.00 \\
\hline $\mathrm{C} 16-\mathrm{C} 17-\mathrm{C} 18$ & $113.52(17)$ & $\mathrm{C} 16-\mathrm{C} 15-\mathrm{H} 15 \mathrm{~A}$ & 109.00 \\
\hline
\end{tabular}




\begin{tabular}{|c|c|c|c|}
\hline $\mathrm{N} 12-\mathrm{C} 18-\mathrm{C} 17$ & $116.96(16)$ & $\mathrm{C} 16-\mathrm{C} 15-\mathrm{H} 15 \mathrm{~B}$ & 109.00 \\
\hline $\mathrm{O} 19-\mathrm{C} 18-\mathrm{N} 12$ & $122.76(16)$ & $\mathrm{H} 15 \mathrm{~A}-\mathrm{C} 15-\mathrm{H} 15 \mathrm{~B}$ & 108.00 \\
\hline $\mathrm{O} 19-\mathrm{C} 18-\mathrm{C} 17$ & $120.27(16)$ & $\mathrm{C} 15-\mathrm{C} 16-\mathrm{H} 16 \mathrm{~A}$ & 109.00 \\
\hline $\mathrm{N} 1-\mathrm{C} 2-\mathrm{H} 2$ & 109.00 & $\mathrm{C} 15-\mathrm{C} 16-\mathrm{H} 16 \mathrm{~B}$ & 109.00 \\
\hline $\mathrm{C} 3-\mathrm{C} 2-\mathrm{H} 2$ & 109.00 & $\mathrm{C} 17-\mathrm{C} 16-\mathrm{H} 16 \mathrm{~A}$ & 109.00 \\
\hline $\mathrm{C} 11-\mathrm{C} 2-\mathrm{H} 2$ & 109.00 & $\mathrm{C} 17-\mathrm{C} 16-\mathrm{H} 16 \mathrm{~B}$ & 109.00 \\
\hline $\mathrm{C} 2-\mathrm{C} 3-\mathrm{H} 3 \mathrm{~A}$ & 110.00 & $\mathrm{H} 16 \mathrm{~A}-\mathrm{C} 16-\mathrm{H} 16 \mathrm{~B}$ & 108.00 \\
\hline $\mathrm{C} 2-\mathrm{C} 3-\mathrm{H} 3 \mathrm{~B}$ & 110.00 & $\mathrm{C} 16-\mathrm{C} 17-\mathrm{H} 17 \mathrm{~A}$ & 109.00 \\
\hline $\mathrm{C} 4-\mathrm{C} 3-\mathrm{H} 3 \mathrm{~A}$ & 110.00 & $\mathrm{C} 16-\mathrm{C} 17-\mathrm{H} 17 \mathrm{~B}$ & 109.00 \\
\hline $\mathrm{C} 4-\mathrm{C} 3-\mathrm{H} 3 \mathrm{~B}$ & 110.00 & $\mathrm{C} 18-\mathrm{C} 17-\mathrm{H} 17 \mathrm{~A}$ & 109.00 \\
\hline $\mathrm{H} 3 \mathrm{~A}-\mathrm{C} 3-\mathrm{H} 3 \mathrm{~B}$ & 108.00 & $\mathrm{C} 18-\mathrm{C} 17-\mathrm{H} 17 \mathrm{~B}$ & 109.00 \\
\hline $\mathrm{N} 12-\mathrm{C} 4-\mathrm{H} 4$ & 107.00 & $\mathrm{H} 17 \mathrm{~A}-\mathrm{C} 17-\mathrm{H} 17 \mathrm{~B}$ & 108.00 \\
\hline $\mathrm{C} 3-\mathrm{C} 4-\mathrm{H} 4$ & 107.00 & & \\
\hline $\mathrm{C} 10-\mathrm{N} 1-\mathrm{C} 2-\mathrm{C} 3$ & $-44.6(2)$ & $\mathrm{C} 3-\mathrm{C} 4-\mathrm{C} 5-\mathrm{C} 6$ & $-160.41(16)$ \\
\hline $\mathrm{C} 10-\mathrm{N} 1-\mathrm{C} 2-\mathrm{C} 11$ & $-168.08(16)$ & $\mathrm{C} 3-\mathrm{C} 4-\mathrm{C} 5-\mathrm{C} 10$ & $22.4(2)$ \\
\hline $\mathrm{C} 2-\mathrm{N} 1-\mathrm{C} 10-\mathrm{C} 5$ & $16.6(2)$ & $\mathrm{C} 4-\mathrm{C} 5-\mathrm{C} 6-\mathrm{C} 7$ & $-176.99(19)$ \\
\hline $\mathrm{C} 2-\mathrm{N} 1-\mathrm{C} 10-\mathrm{C} 9$ & $-165.36(16)$ & $\mathrm{C} 10-\mathrm{C} 5-\mathrm{C} 6-\mathrm{C} 7$ & $0.2(3)$ \\
\hline $\mathrm{C} 13-\mathrm{N} 12-\mathrm{C} 4-\mathrm{C} 3$ & $56.10(18)$ & $\mathrm{C} 4-\mathrm{C} 5-\mathrm{C} 10-\mathrm{N} 1$ & $-4.7(2)$ \\
\hline $\mathrm{C} 13-\mathrm{N} 12-\mathrm{C} 4-\mathrm{C} 5$ & $-69.81(17)$ & $\mathrm{C} 4-\mathrm{C} 5-\mathrm{C} 10-\mathrm{C} 9$ & $177.21(15)$ \\
\hline $\mathrm{C} 18-\mathrm{N} 12-\mathrm{C} 4-\mathrm{C} 3$ & $-127.62(15)$ & $\mathrm{C} 6-\mathrm{C} 5-\mathrm{C} 10-\mathrm{N} 1$ & $178.05(16)$ \\
\hline $\mathrm{C} 18-\mathrm{N} 12-\mathrm{C} 4-\mathrm{C} 5$ & $106.48(16)$ & $\mathrm{C} 6-\mathrm{C} 5-\mathrm{C} 10-\mathrm{C} 9$ & $0.0(2)$ \\
\hline $\mathrm{C} 4-\mathrm{N} 12-\mathrm{C} 13-\mathrm{C} 14$ & $-113.04(16)$ & $\mathrm{C} 5-\mathrm{C} 6-\mathrm{C} 7-\mathrm{C} 8$ & $-0.5(3)$ \\
\hline $\mathrm{C} 18-\mathrm{N} 12-\mathrm{C} 13-\mathrm{C} 14$ & $70.8(2)$ & $\mathrm{C} 6-\mathrm{C} 7-\mathrm{C} 8-\mathrm{C} 9$ & $0.5(3)$ \\
\hline $\mathrm{C} 4-\mathrm{N} 12-\mathrm{C} 18-\mathrm{O} 19$ & $1.8(2)$ & $\mathrm{C} 7-\mathrm{C} 8-\mathrm{C} 9-\mathrm{C} 10$ & $-0.3(3)$ \\
\hline $\mathrm{C} 4-\mathrm{N} 12-\mathrm{C} 18-\mathrm{C} 17$ & $-179.65(14)$ & $\mathrm{C} 8-\mathrm{C} 9-\mathrm{C} 10-\mathrm{N} 1$ & $-178.00(17)$ \\
\hline $\mathrm{C} 13-\mathrm{N} 12-\mathrm{C} 18-\mathrm{O} 19$ & $177.93(15)$ & $\mathrm{C} 8-\mathrm{C} 9-\mathrm{C} 10-\mathrm{C} 5$ & $0.1(3)$ \\
\hline $\mathrm{C} 13-\mathrm{N} 12-\mathrm{C} 18-\mathrm{C} 17$ & $-3.5(2)$ & $\mathrm{N} 12-\mathrm{C} 13-\mathrm{C} 14-\mathrm{C} 15$ & $-79.6(2)$ \\
\hline $\mathrm{N} 1-\mathrm{C} 2-\mathrm{C} 3-\mathrm{C} 4$ & $60.77(18)$ & $\mathrm{C} 13-\mathrm{C} 14-\mathrm{C} 15-\mathrm{C} 16$ & $56.7(3)$ \\
\hline $\mathrm{C} 11-\mathrm{C} 2-\mathrm{C} 3-\mathrm{C} 4$ & $-177.44(16)$ & $\mathrm{C} 14-\mathrm{C} 15-\mathrm{C} 16-\mathrm{C} 17$ & $-59.5(3)$ \\
\hline $\mathrm{C} 2-\mathrm{C} 3-\mathrm{C} 4-\mathrm{N} 12$ & $-176.30(13)$ & $\mathrm{C} 15-\mathrm{C} 16-\mathrm{C} 17-\mathrm{C} 18$ & $83.3(2)$ \\
\hline $\mathrm{C} 2-\mathrm{C} 3-\mathrm{C} 4-\mathrm{C} 5$ & $-49.92(18)$ & $\mathrm{C} 16-\mathrm{C} 17-\mathrm{C} 18-\mathrm{O} 19$ & $112.4(2)$ \\
\hline $\mathrm{N} 12-\mathrm{C} 4-\mathrm{C} 5-\mathrm{C} 6$ & $-33.7(2)$ & $\mathrm{C} 16-\mathrm{C} 17-\mathrm{C} 18-\mathrm{N} 12$ & $-66.2(2)$ \\
\hline $\mathrm{N} 12-\mathrm{C} 4-\mathrm{C} 5-\mathrm{C} 10$ & $149.10(14)$ & & \\
\hline
\end{tabular}

Hydrogen-bond geometry $\left(\AA,{ }^{\circ}\right)$

\begin{tabular}{lllll}
\hline$D-\mathrm{H} \cdots A$ & $D-\mathrm{H}$ & $\mathrm{H} \cdots A$ & $D \cdots A$ & $D-\mathrm{H} \cdots A$ \\
\hline $\mathrm{N} 1-\mathrm{H} 1 \cdots \mathrm{O} 19^{\mathrm{i}}$ & 0.86 & 2.40 & $2.988(2)$ & 126 \\
$\mathrm{C} 14-\mathrm{H} 14 A \cdots \mathrm{O} 19^{\mathrm{ii}}$ & 0.97 & 2.57 & $3.320(2)$ & 134 \\
\hline
\end{tabular}

Symmetry codes: (i) $x+1 / 2,-y+3 / 2, z-1 / 2$; (ii) $-x+1 / 2, y+1 / 2,-z+1 / 2$. 